Revista Brasileira de Agricultura Irrigada v.13, nº.4, p. 3540 - 3555, 2019

ISSN 1982-7679 (On-line)

Fortaleza, CE, INOVAGRI - http://www.inovagri.org.br

DOI: $10.7127 /$ rbai.v13n4001078

Protocolo 1078.19 - 19/03/2019 Aprovado em 28/07/2019

\title{
ZONAS HOMOGÊNEAS DE EVAPOTRANSPIRAÇÃO DE REFERÊNCIA PARA O NORTE E NOROESTE DE MINAS GERAIS
}

\author{
Tarlei Aparecido Santos ${ }^{1}$, Marcelo Rossi Vicente ${ }^{2}$, Caio Vinicius Leite ${ }^{3}$, Ronaldo Medeiros dos \\ Santos ${ }^{4}$, José Luís Teixeira de Souza ${ }^{5}$
}

\begin{abstract}
RESUMO
Considerando a importância econômica da produção irrigada para as regiões Noroeste e Norte do Estado de Minas Gerais e a importância do uso racional da água para manutenção da agricultura irrigada, este trabalho teve como objetivo mapear zonas homogêneas de evapotranspiração de referência $\left(\mathrm{ET}_{0}\right)$ nas mesorregiões supracitadas. Foi utilizada a rede de estações meteorológicas convencionais, situadas dentro e ao redor da área de estudo, totalizando 34 estações utilizadas. Os dados meteorológicos, necessários para o zoneamento, foram obtidos de uma série histórica de 32 anos (1985-2016). Foram estimados os valores médios de ET $_{0}$ pelo método de Penman-Monteith FAO e o Balanço Hídrico Climatológico (BHC). O método utilizado para interpolação dos dados foi do tipo Krigagem ordinária. Para a validação das zonas homogêneas, foram aplicados diferentes indicadores estatísticos, que correlacionaram os dados observados com os dados estimados. Com base no BHC, foram considerados críticos os meses de julho, agosto, setembro e outubro. Foram identificadas 5 zonas homogêneas de $\mathrm{ET}_{0}$. As médias de $\mathrm{ET}_{0}$ preditas pela Krigagem ordinária foram similares às observadas (medidas) pelas estações meteorológicas convencionais, uma vez que os indicadores estatísticos se mostraram satisfatórios.
\end{abstract}

Palavras-chave: krigagem ordinária, geoestatística, irrigação.

\section{REFERENCE EVAPOTRANSPIRATION ZONES FOR THE NORTH AND NORTHWEST REGIONS OF THE MINAS GERAIS STATE}

\footnotetext{
${ }^{1}$ Engenheiro Florestal, Mestrando em Ciências Geodésicas e Tecnologias da Geoinformação. Universidade Federal de Pernambuco - Campus Recife, Recife, PE. E-mail: tarleyengflorestal@gmail.com

${ }^{2}$ Engenheiro Agrônomo, Doutor, Docente do Instituto Federal do Norte de Minas - Campus Salinas, Salinas, MG. Email: marcelo.vicente@ifnmg.edu.br

${ }^{3}$ Engenheiro Agrícola e Ambiental, Mestre, Docente do Instituto Federal de Brasília - Campus Planaltina, Planaltina, DF. E-mail: caio.leite@ifb.edu.br

${ }^{4}$ Engenheiro Agrimensor, Doutor, Docente do Instituto Federal do Norte de Minas - Campus Salinas, Salinas, MG. Email: ronaldo.medeiros@ifnmg.edu.br

${ }^{5}$ Graduando em Engenharia Florestal, Bolsista PIBIC CNPq, Instituto Federal do Norte de Minas - Campus Salinas, Salinas, MG. E-mail: jose.engeflor@gmail.com
} 


\begin{abstract}
Considering the economic importance of irrigated crop production for the Northwest and North regions of Minas Gerais State and the relevance of the rational water use for maintenance of irrigated agriculture, this work aimed to map homogeneous zones of reference evapotranspiration $\left(\mathrm{ET}_{0}\right)$ at those regions. The weather stations network around the study area was composed by 34 stations and the data were obtained through the BDMEP / INMET system platform, from a 32-year historical series (1985-2016). The mean $\mathrm{ET}_{0}$ values were estimated using the FAO Penman-Monteith method and the water balance. The ordinary krigging technique was used for data interpolation. Different statistical indicators were applied to validate the homogeneous zones, correlating the observed data with the estimated one. July, August, September and October were considered critical months to the water balance. Five homogeneous $\mathrm{ET}_{0}$ zones were identified and the $\mathrm{ET}_{0}$ averages predicted by ordinary kriging were similar to those observed (measured) by the weather stations, once the statistical indicators were satisfactory.
\end{abstract}

Keywords: ordinary kriging, geostatistics, irrigation.

\section{INTRODUÇÃO}

Diante do aumento crescente da escassez hídrica que vem ocorrendo em várias regiões do mundo, a quantificação da evapotranspiração é de fundamental importância para o desenvolvimento sustentável da agricultura irrigada (VICENTE et al., 2015).

A evapotranspiração tem grande influência na perda de água nas bacias hidrográficas e está estreitamente relacionada com a dinâmica da umidade do solo, a recarga dos aquíferos e ao escoamento superficial. Portanto o entendimento das variações da evapotranspiração é de grande relevância em estudos hidrológicos regionais (LIANG et al., 2009). Também desempenha um importante papel na gestão de bacias hidrográficas, no manejo da agricultura irrigada e em estudos relacionados a modelagem meteorológica e hidrológica (BEZERRA et al., 2008).

Controlada pelo balanço de energia, a evapotranspiração depende da demanda atmosférica, da disponibilidade de água presente no solo e das características fisiológicas das plantas (SANCHES et al., 2015). A evapotranspiração pode ser medida diretamente ou estimada por modelos físicomatemáticos ou empíricos.

O método de Penman-Monteith-FAO é o mais utilizado no mundo para estimar a $\mathrm{ET}_{0}$ (PEREIRA et al., 2015). Esse método requer uma quantidade expressiva de variáveis climáticas, o que pode dificultar a sua utilização por pequenos e médios produtores agrícolas, que não dispõe das informações meteorológicas e pelo elevado custo de aquisição de estações meteorológicas (VICENTE et al., 2014). Diante de tais limitações, a disponibilização da distribuição espacial mensal da $\mathrm{ET}_{0}$ se apresenta como uma alternativa viável, que possibilitaria aos irrigantes, pequenos e médios, uma informação prontamente utilizável para o uso mais eficiente da irrigação e, consequentemente, de água e energia (SILVA JUNIOR, 2017).

Como o INMET opera aproximadamente 900 estações de superfície, dentre convencionais e automáticas (VIANELLO; ALVES, 2012), o uso de ferramentas geoestatísticas para a espacialização de dados climáticos é uma alternativa que pode proporcionar maior acesso às informações de $\mathrm{ET}_{0}$ pelos irrigantes e usuários em geral.

Um dos principais métodos de interpolação de dados é a krigagem ordinária, que usa a dependência espacial entre amostras vizinhas, expressa no semivariograma, para estimar valores em qualquer posição dentro do espaço analisado, ao qual o modelo do semivariograma foi ajustado, sem tendência e com variância mínima (MOTOMIYA et al., 2006). 
Mancosu et al. (2014), analisando três técnicas de interpolação, quando estabeleceram procedimentos para o desenvolvimento de mapas de zonas de evapotranspiração de referência, indicaram a krigagem como a melhor para a espacialização da $\mathrm{ET}_{0}$.

Vila Nova et al. (2012), realizaram um estudo que avaliava a qualidade dos valores de $\mathrm{ET}_{0}$ médios mensais interpolados geoespacialmente a partir do processo de krigagem, no Sul de Minas Gerais, por meio do qual constataram que o referido método pode ser aplicado para elaboração de mapas de $\mathrm{ET}_{0}$ em regiões com escassez de dados hidrometeorológicos, desde que os procedimentos para seleção das estações meteorológicas utilizadas e validação dos resultados sejam considerados.

Já Silva Junior (2017), utilizando a krigagem, realizou o mapeamento de zonas homogêneas de evapotranspiração de referência para região Noroeste Paulista, como ferramenta para o manejo sustentável da irrigação.

$\mathrm{Na}$ Califórnia, EUA, destaca-se o Sistema de Informação de Manejo da Irrigação da Califórnia (California Irrigation Management Information System - CIMIS), desenvolvido pelo Departamento de Recursos Hídricos da Califórnia (California Department of Water Resources - DWR) e pela Universidade da Califórnia, que gerencia uma rede de mais de 145 estações meteorológicas automáticas em 18 zonas homogêneas de evapotranspiração de referência.

Diante do exposto, o trabalho teve por objetivo mapear zonas homogêneas de evapotranspiração de referência $\left(\mathrm{ET}_{0}\right)$ nas mesorregiões do Norte e Noroeste do estado de Minas Gerais, como forma de disponibilizar aos irrigantes da região uma informação imprescindível à gestão eficiente dos recursos hídricos, energéticos e financeiros.

\section{MATERIAIS E MÉTODOS}

\section{Área de estudo}

A área de estudo compreendeu as mesorregiões do Norte e Noroeste do estado de Minas Gerais, conforme ilustrado na Figura 1. Juntas, essas compreendem uma superfície de aproximadamente $190.801 \mathrm{~km}^{2}$, situada entre os paralelos $14^{\circ}$ e $19^{\circ}$ de latitude sul e entre os meridianos $47^{\circ}$ e $41^{\circ}$ a oeste de Greenwich. 


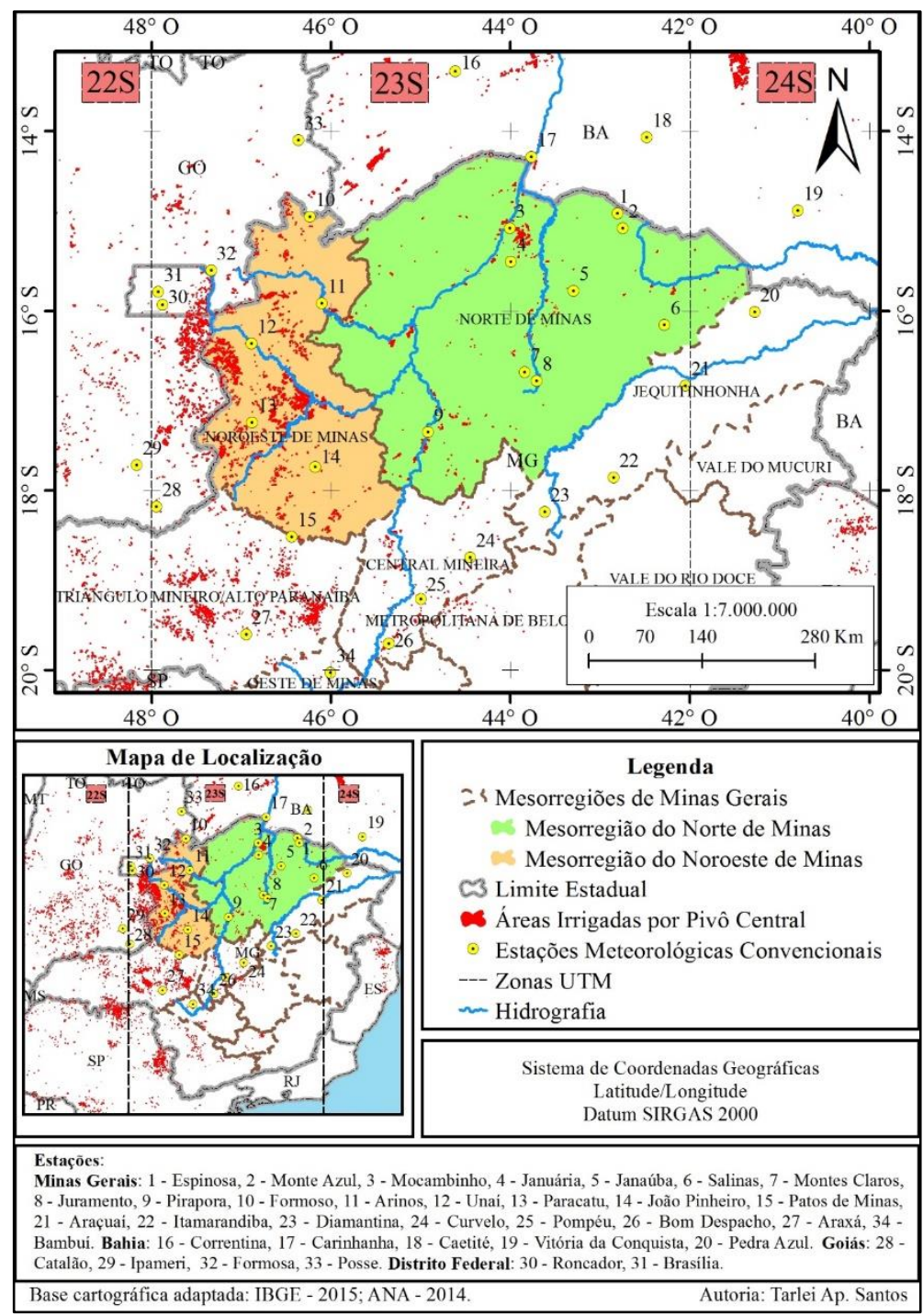

Figura 1. Mapa de Localização da mesorregião do Norte e Noroeste do estado de Minas Gerais. Fonte: Adaptado da base cartográfica IBGE, 2015 (Divisão das mesorregiões e microrregiões do Brasil) e da base cartográfica ANA, 2014 (Áreas irrigadas por pivô central no Brasil em 2014).

A mesorregião do Norte de Minas Gerais é composta por sete microrregiões: Bocaiúva, Grão Mogol, Janaúba, Januária, Montes Claros, Salinas e Pirapora. Abrange 89 municípios e ocupa uma área de aproximadamente $128.451 \quad \mathrm{~km}^{2}$, com temperatura média anual de $24,08{ }^{\circ} \mathrm{C}$ e precipitação média anual de $900 \mathrm{~mm}$ (INMET, 2018). Já a mesorregião do Noroeste de Minas Gerais é formada por duas microrregiões: Unaí e Paracatu, com uma área aproximada de $62.351 \mathrm{Km}^{2}$, abrangendo 19 municípios. Possui temperatura e precipitação anual média de $23,92{ }^{\circ} \mathrm{C}$ e $1334 \mathrm{~mm}$, respectivamente (INMET, 2018).
Foi utilizada uma rede de estações meteorológicas localizadas dentro da área de interesse, composta por 14 estações convencionais; todas sob administração do INMET, sendo cinco estações localizadas na mesorregião Noroeste e nove estações localizadas na mesorregião Norte. Ademais, foram selecionadas no entorno da área de interesse outras 21 estações, com o intuito de se minimizar o efeito de borda. Neste caso, foram selecionadas estações nos estados da Bahia (4), Goiás (4), no Distrito Federal (2) e 11 estações no estado de Minas Gerais, entre o oeste e o sul da área de interesse. No total, foram utilizadas 35 estações (Figura 1). 


\section{Rede das Estações Meteorológicas}

Os dados meteorológicos necessários para o mapeamento das zonas homogêneas $\mathrm{ET}_{0}$ foram obtidos através da plataforma do sistema BDMEP (Banco de Dados Meteorológicos para Ensino e Pesquisa) (INMET, 2017). Foram obtidas informações de uma série histórica de 32 anos (1985-2016), em uma escala mensal, que continha informações sobre: velocidade média do vento; insolação total; precipitação total; temperatura máxima média; temperatura mínima média e; umidade relativa média.

\section{Evapotranspiração de referência e balanço hídrico climatológico (BHC)}

A partir das variáveis meteorológicas, foi estimada a evapotranspiração de referência $\left(\mathrm{ET}_{0}\right)$, em escala mensal, pela metodologia proposta por Penman-Monteith - FAO (ALLEN et al., 1998). O método utilizado para calcular o Balanço Hídrico Climatológico (BHC) foi o proposto por Thornthwaite e Mather (1955), com o auxílio da ferramenta "BHnorm", elaborado em planilha por Rolim et al. (1998). Para a estimativa do BHC foram necessárias as variáveis de evapotranspiração de referência $\left(\mathrm{ET}_{0}\right)$, precipitação e capacidade de água disponível no solo (CAD). No caso desta última, adotou-se um valor de referência de $100 \mathrm{~mm}$, conforme recomendado por Vianello e Alves (2012).

$\mathrm{O}$ BHC identificou os meses mais críticos para o manejo da irrigação, a partir dos quais foi possível identificar e zonear as áreas homogêneas quanto à distribuição da $\mathrm{ET}_{0}$.

\section{Procedimentos para elaboração dos mapas climáticos}

A base cartográfica foi obtida gratuitamente no formato shapefile (.shp), compreendendo os mapas temáticos vetoriais das meso e microrregiões do Norte e Noroeste de Minas Gerais, além dos limites municipais e estaduais das unidades da federação disponibilizada no site do IBGE na escala 1:1.000.000. A base hidrográfica e a área de pivôs centrais de irrigação, para ano de 2014, foram obtidas através do site da Agência Nacional de Águas (ANA) na escala 1:1.000.000 e 1:250.000, respectivamente (ANA; EMBRAPA, 2016).

Utilizou softwares livres de sistema de informação geográfica para organização/gerenciamento dos dados geográficos. Da base cartográfica, foram selecionados apenas os dados contidos dentro da área de interesse, para o estabelecimento do mapa base para interpolação dos resultados. Para a modelagem dos semivariogramas experimentais foi utilizado o software $\mathrm{GS}^{+}$ (Gamma Design Software, 2016), versão "Demo".

\section{Método de interpolação e ajuste de modelos à semivariogramas experimentais}

O método utilizado para interpolação dos dados foi a Krigagem ordinária, uma vez que a krigagem apresenta estimativas não tendenciosas e a mínima variância associada ao valor estimado (PIRES et al., 2016). Utilizou-se o software $\mathrm{GS}^{+}$, versão Demo, para o ajuste automático de modelos teóricos a semivariogramas experimentais dos dados.

O software possui como critério para avaliar o melhor ajuste, o valor do coeficiente de determinação $\left(\mathrm{R}^{2}\right)$ e o menor valor para soma de quadrados de resíduos (SQres). Ajustando-se as informações para o tipo (exponencial, linear, esférico, gaussiano) e os parâmetros (alcance da dependência espacial, efeito pepita, distância entre o efeito pepita e o patamar) do modelo de semivariograma, foram transferidos, para o software livre de sistema de informação geográfica, os resultados, a partir dos quais foram interpoladas as variáveis e elaborados os mapas de distribuição espacial da $\mathrm{ET}_{0}$ na área de estudo.

\section{Interpolação e definição das zonas homogêneas \\ Para interpolação dos dados, o} procedimento utilizado foi o mesmo adotado por Silva Junior (2017), onde no software livre de sistema de informação geográfica, através de ferramentas de geoestatística, 
selecionaram-se as opções krigagem ordinária e modelo Gaussiano para o ajuste de semivariogramas experimentais. Ao final, separaram-se as classes de $\mathrm{ET}_{0}$ em intervalos padronizados de $0,5 \mathrm{~mm} \mathrm{dia}{ }^{-1}$.

As zonas homogêneas de $\mathrm{ET}_{0}$ foram definidas a partir dos resultados obtidos do balanço hídrico climatológico, onde os meses que registraram os maiores valores de déficit hídrico (mais críticos para o manejo da irrigação) serviram como referência para determinação das zonas homogêneas, conforme Silva Junior et al. (2018). A interseção das classes de $\mathrm{ET}_{0}$, padronizadas em intervalos de $0,5 \mathrm{~mm} \mathrm{dia}^{-1}$, para os meses mais críticos, permitiu a visualização das áreas equivalentes. A partir da identificação das áreas equivalentes, foram desenhados semiautomaticamente os polígonos que caracterizaram as zonas homogêneas.

\section{Validação das Zonas Homogêneas}

Para a validação das zonas homogêneas, foram utilizados diferentes indicadores estatísticos que correlacionaram os valores mensais de $\mathrm{ET}_{0}\left(\mathrm{~mm} \mathrm{dia}^{-1}\right)$ observados pelas 14 estações meteorológicas convencionais, localizadas dentro da área de interesse, com os valores médios mensais de $\mathrm{ET}_{0}\left(\mathrm{~mm} \mathrm{dia}^{-1}\right)$, estimados com base na média de cada zona homogênea predita pela Krigagem ordinária, conforme a metodologia proposta por Castro et al. (2010).

Os indicadores utilizados para a validação das zonas homogêneas de $\mathrm{ET}_{0}$ foram: o coeficiente de correlação de Pearson $(\mathrm{r})$; o coeficiente de determinação $\left(\mathrm{R}^{2}\right)$; a raiz do erro médio quadrático (REMQ); o índice de concordância (d) de Willmott (1981); o coeficiente de eficiência de Nash-Sutcliffe (NSE) (NASH; SUTCLIFFE, 1970); o erro médio absoluto (EMA); e o erro médio percentual (EMP) e o índice de confiança (c) proposto por Camargo e Sentelhas (1997).

Os critérios de avaliação do desempenho de modelos quanto ao índice de confiança, estão apresentados na Tabela 1.

Tabela 1. Análise do desempenho do modelo com base no índice de confiança.

\begin{tabular}{cc}
\hline Valor de c & Desempenho \\
\hline$>0,85$ & Ótimo \\
0,76 a 0,85 & Muito bom \\
0,66 a 0,75 & Bom \\
0,61 a 0,65 & Mediano \\
0,51 a 0,60 & Sofrível \\
0,41 a 0,50 & Mau \\
$\leq 0,4$ & Péssimo \\
\hline
\end{tabular}

Fonte: Camargo e Sentelhas (1997).

Foi comparado, dentro de cada zona homogênea, o valor médio de $\mathrm{ET}_{0}$, obtido a partir dos dados medidos nas estações com os valores de $\mathrm{ET}_{0}$ preditos via interpolação por Krigagem.

Após a validação do mapa que representou as zonas homogêneas, foi construída uma tabela relativa aos valores médios mensais $\mathrm{ET}_{0}$ para cada zona.

\section{RESULTADOS E DISCUSSÃO}

\section{Evapotranspiração e Balanço Hídrico Climatológico}

Na Tabela 2 são apresentados os valores médios diários de $\mathrm{ET}_{0}$. Observa-se que a demanda evapotranspirométricas reduziu continuamente entre os meses de janeiro e junho, quando atingiram os menores valores e, posteriormente, aumentou progressivamente até atingir os maiores valores em outubro. Essa tendência é observada em todas as estações do Norte e Noroeste de Minas. 
Tabela 2. Médias da evapotranspiração de referência $\left(\mathrm{ET}_{0}\right)$, calculadas pelo método de PenmanMonteith - FAO.

\begin{tabular}{|c|c|c|c|c|c|c|c|c|c|c|c|c|c|}
\hline \multirow{2}{*}{ Estações } & \multicolumn{13}{|c|}{$\mathrm{ET}_{0}\left(\mathrm{~mm} \mathrm{dia}^{-1}\right)$} \\
\hline & Jan & $\mathrm{Fev}$ & Mar & Abr & Mai & Jun & Jul & Ago & Set & Out & Nov & Dez & Ano \\
\hline Espinos & 4,84 & 5,51 & 5,04 & 4,84 & 4,51 & 4,44 & 4,68 & 6,00 & 6,71 & 6,70 & 5,13 & 4,93 & 5,28 \\
\hline Monte A & 4,94 & 5,31 & 4,90 & 4,83 & 4,50 & 4,43 & 4,73 & 5,69 & 6,48 & 6,37 & 5,08 & 4,62 & 5,16 \\
\hline Mocambin & 4,39 & 4,70 & 4,42 & 4,42 & 3,91 & 3,68 & 3,84 & 4,31 & 5,09 & 5,15 & 4,49 & 4,21 & 4,38 \\
\hline Januária & 4,67 & 5,11 & 4,62 & 4,55 & 4,09 & 3,92 & 4,38 & 5,39 & 6,27 & 6,34 & 4,75 & 4,48 & 4,88 \\
\hline Janaúb & 4,61 & 5,25 & 4,63 & 4,54 & 4,09 & 3,86 & 3,99 & 4,82 & 5,26 & 5,27 & 4,48 & 4,20 & 4,58 \\
\hline Salin & 4,55 & 4,99 & 4,36 & 3,79 & 3,19 & 2,93 & 3,29 & 4,19 & 4,98 & 5,33 & 4,36 & 4,33 & 4,19 \\
\hline Montes Cla & 4,80 & 5,17 & 4,56 & 4,27 & 3,74 & 3,46 & 3,79 & 4,81 & 5,66 & 5,64 & 4,65 & 4,40 & 4,58 \\
\hline Jurame & 4,39 & 4,69 & 4,10 & 3,78 & 3,29 & 3,05 & 3,15 & 4,10 & 4,86 & 5,01 & 4,31 & 4,06 & 4,07 \\
\hline Pirap & 4,51 & 4,89 & 4,27 & 4,16 & 3,49 & 3,18 & 3,42 & 4,46 & 5,45 & 5,61 & 4,63 & 4,37 & 4,37 \\
\hline Form & 4,16 & 4,46 & 4,13 & 4,10 & 3,88 & 3,68 & 4,03 & 4,74 & 5,22 & & 4,08 & 3,87 & 4,30 \\
\hline $1 G$ & 3,92 & 4,08 & 3,73 & 3,50 & 3,01 & 2,75 & 3,16 & 3,76 & 4,35 & 4,49 & 3,83 & 3,55 & 3,68 \\
\hline Una & 4,12 & 4,06 & 3,74 & 3,50 & 3,08 & 2,89 & 3,14 & 3,89 & 4,68 & 4,77 & 3,87 & 3,54 & 3,77 \\
\hline Para & 4,34 & 4,58 & 4,10 & 3,89 & 3,40 & 3,17 & 3,62 & 4,59 & 5,36 & 5,35 & 4,49 & 4,19 & 4,26 \\
\hline João Pinheiro - MG & 3,86 & 4,33 & 3,97 & 3,83 & 3,35 & 3,10 & 3,39 & 4,00 & 4,42 & 4,49 & 4,04 & 3,87 & 3,89 \\
\hline de Miv & & & & & & & 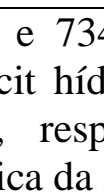 & & & & & & \\
\hline
\end{tabular}
valores acumulados de chuvas $(667 \mathrm{~mm}, 734$

Tabela 3. Resumo anual dos parâmetros precipitação $(\mathrm{P})$, evapotranspiração de referência $\left(\mathrm{ET}_{0}\right)$, déficit hídrico (DEF) e excedente hídrico (EXC) para as regiões Norte e Noroeste de Minas Gerais.

\begin{tabular}{ccccc}
\hline Estações & $\mathrm{P}(\mathrm{mm})$ & $\mathrm{ET}_{0}(\mathrm{~mm})$ & $\mathrm{DEF}(\mathrm{mm})$ & $\mathrm{EXC}(\mathrm{mm})$ \\
\hline Espinosa - MG & 667 & 1.925 & 1.258 & - \\
Monte Azul - MG & 734 & 1.882 & 1.148 & - \\
Mocambinho - MG & 830 & 1.599 & 769 & - \\
Januária - MG & 942 & 1.782 & 891 & 52 \\
Janaúba - MG & 734 & 1.671 & 938 & - \\
Salinas - MG & 830 & 1.528 & 698 & 1 \\
Montes Claros - MG & 1.015 & 1.670 & 770 & 115 \\
Juramento - MG & 902 & 1.482 & 626 & 45 \\
Pirapora - MG & 1.060 & 1.593 & 684 & 151 \\
Formoso - MG & 1.224 & 1.568 & 613 & 270 \\
Arinos - MG & 1.116 & 1.341 & 493 & 268 \\
Unaí - MG & 1.341 & 1.376 & 445 & 409 \\
Paracatu - MG & 1.421 & 1.553 & 575 & 443 \\
João Pinheiro - MG & 1.338 & 1.418 & 496 & 416 \\
\hline As estações localizadas na mesorregião & evapotranspirométricas (média de 1.599 mm \\
do Norte de Minas Gerais apresentaram os & ano-1). Os menores valores, por sua vez, \\
maiores valores de deficiência hídrica (média & ocorreram nas estações localizadas na \\
de $\quad$ 865 mm ano ${ }^{-1}$ ) e de demanda & mesorregião do Noroeste de Minas Gerais;
\end{tabular}


com média de $524 \mathrm{~mm}^{-1}$ para a deficiência hídrica e de $1.451 \mathrm{~mm}^{\text {ano }}{ }^{-1}$ para a demanda evapotranspirométricas. As estações dessa mesorregião também apresentaram as maiores taxas de precipitação (média de $1.288 \mathrm{~mm}$ ano $^{-1}$ ). A mesorregião Noroeste de Minas apresentou melhores condições climáticas para o desenvolvimento da agricultura de sequeiro, comparada à mesorregião do Norte de Minas.

Analisando o balanço hídrico climatológico (Figura 2), observou-se que os meses de julho, agosto, setembro e outubro foram os mais críticos para o manejo da irrigação, por apresentarem os maiores valores de $\mathrm{ET}_{0}$ e deficiência hídrica (Figura 2). A identificação dos meses de deficiência hídrica mais acentuada (julho, agosto, setembro e outubro) serviu de base para o zoneamento das zonas homogêneas de evapotranspiração de referência.
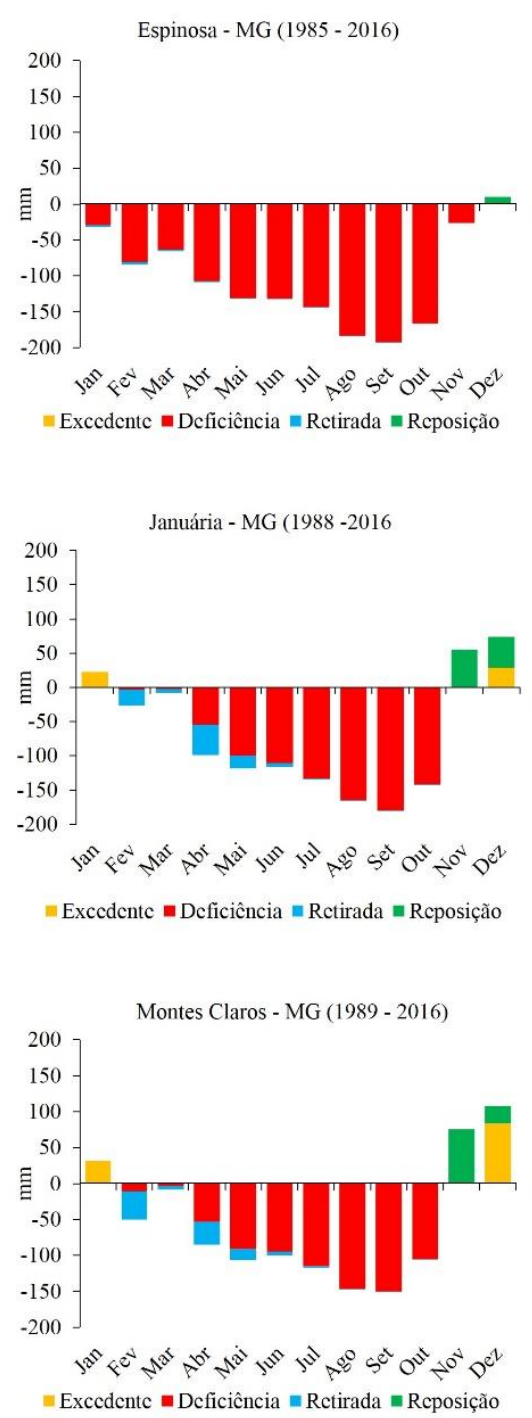
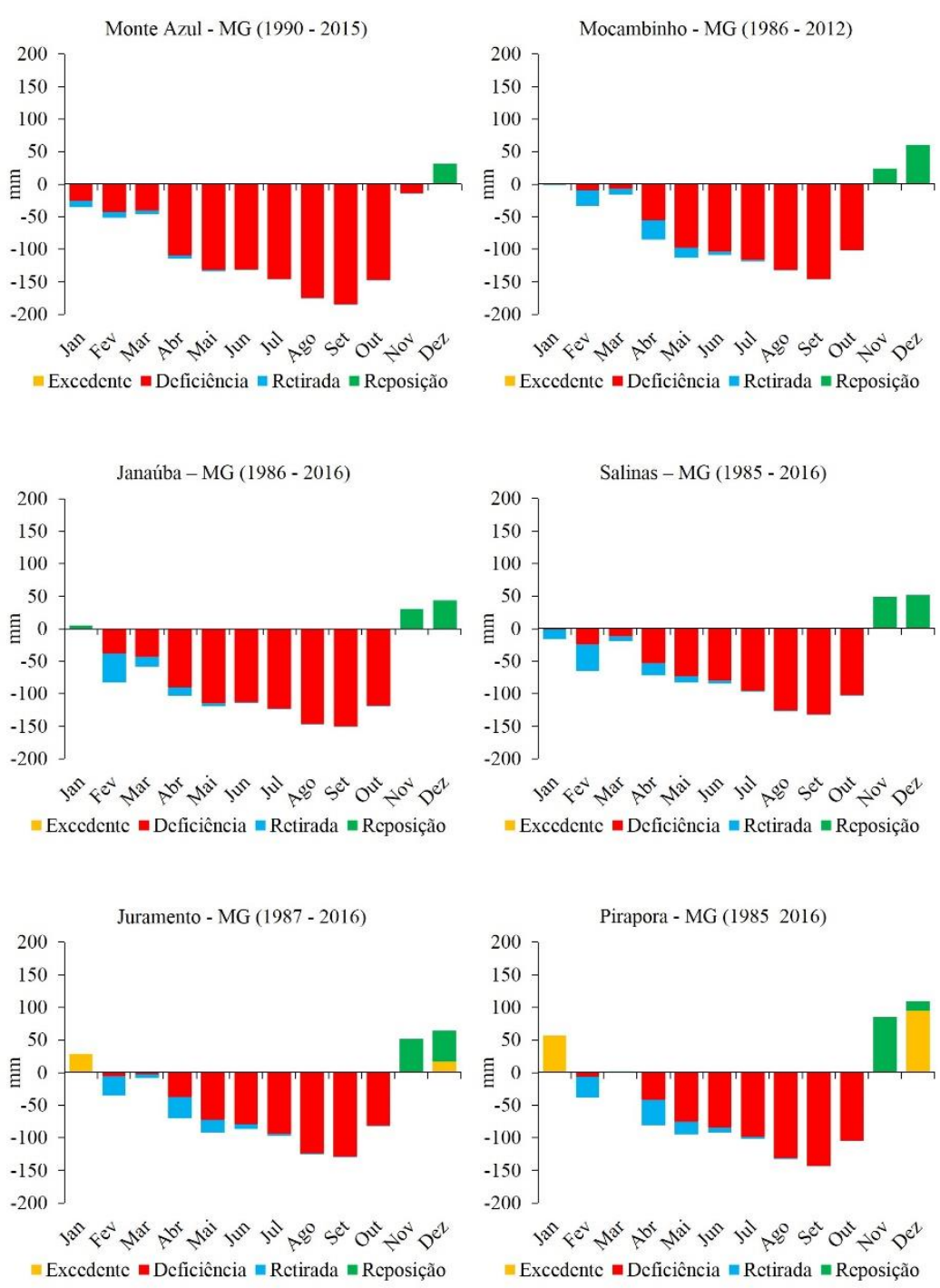

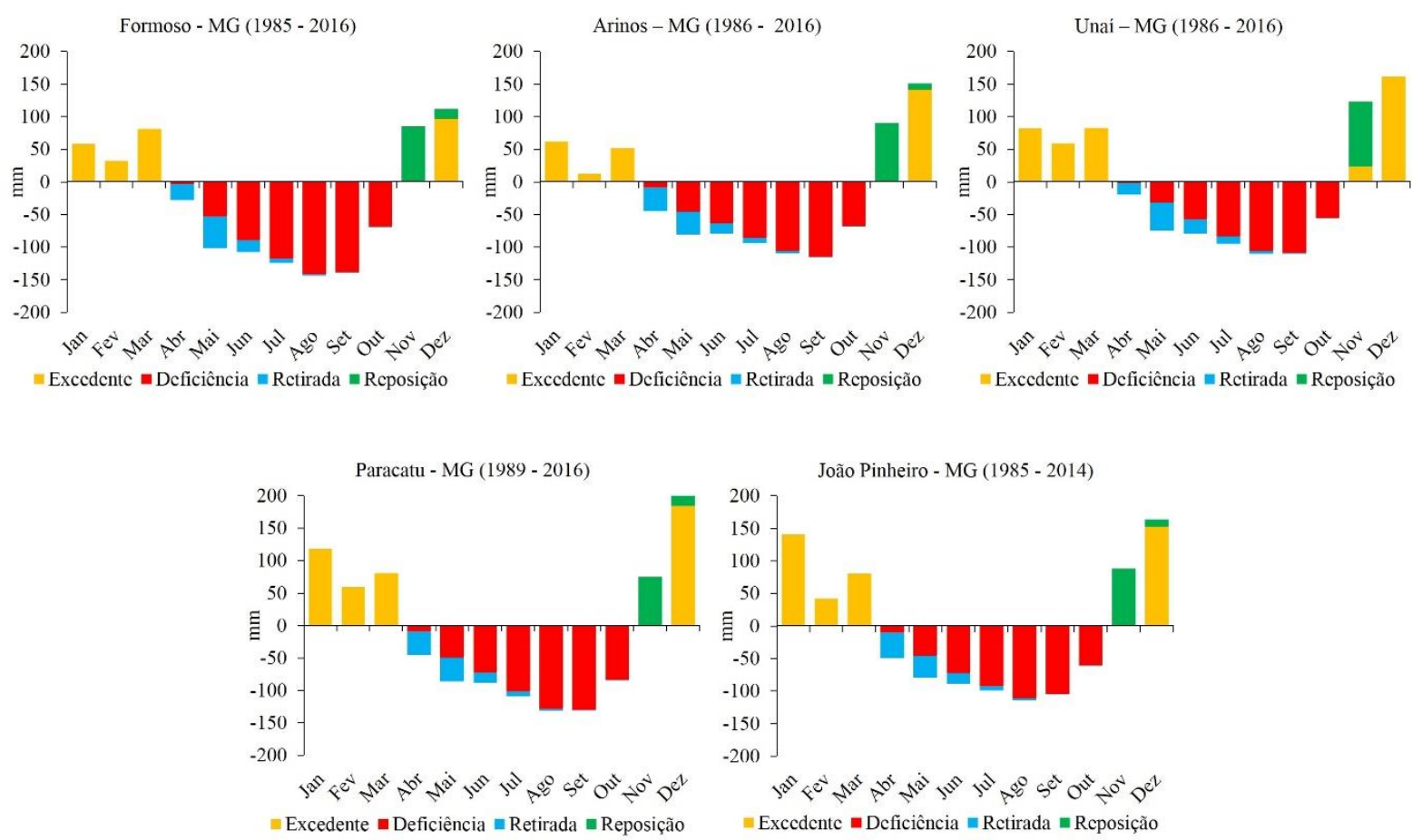

Figura 2. Balanço Hídrico Climatológico (BHC), para o período de 1985 a 2016, utilizando os dados da rede estações meteorológicas convencionais do INMET, para a região Norte e Noroeste, do estado de Minas Gerais.

\section{Distribuição espacial da ET 0}

Os ajustes dos modelos dos semivariogramas experimentais, para interpolação de dados da $\mathrm{ET}_{0}$ por Krigagem ordinária, foram do tipo Gaussiano, adotandose como critério de escolha os melhores valores dos índices estatísticos "Coeficiente de determinação" ( $\left.\mathrm{R}^{2}\right)$ e "soma de quadrados de resíduos" (SQres).
Na Figura 3 observa-se a distribuição espacial da $\mathrm{ET}_{0}$. Observa-se que a demanda evapotranspirométricas reduz continuamente entre meses de janeiro e junho, quando atinge o menor valor da $\mathrm{ET}_{0}$ (média de $3,35 \mathrm{~mm}$ dia $\left.{ }^{1}\right) \quad \mathrm{e}$ posteriormente, aumenta progressivamente até atingir o maior valor em outubro (média de 5,35 $\mathrm{mm} \mathrm{dia}^{-1}$ ). 


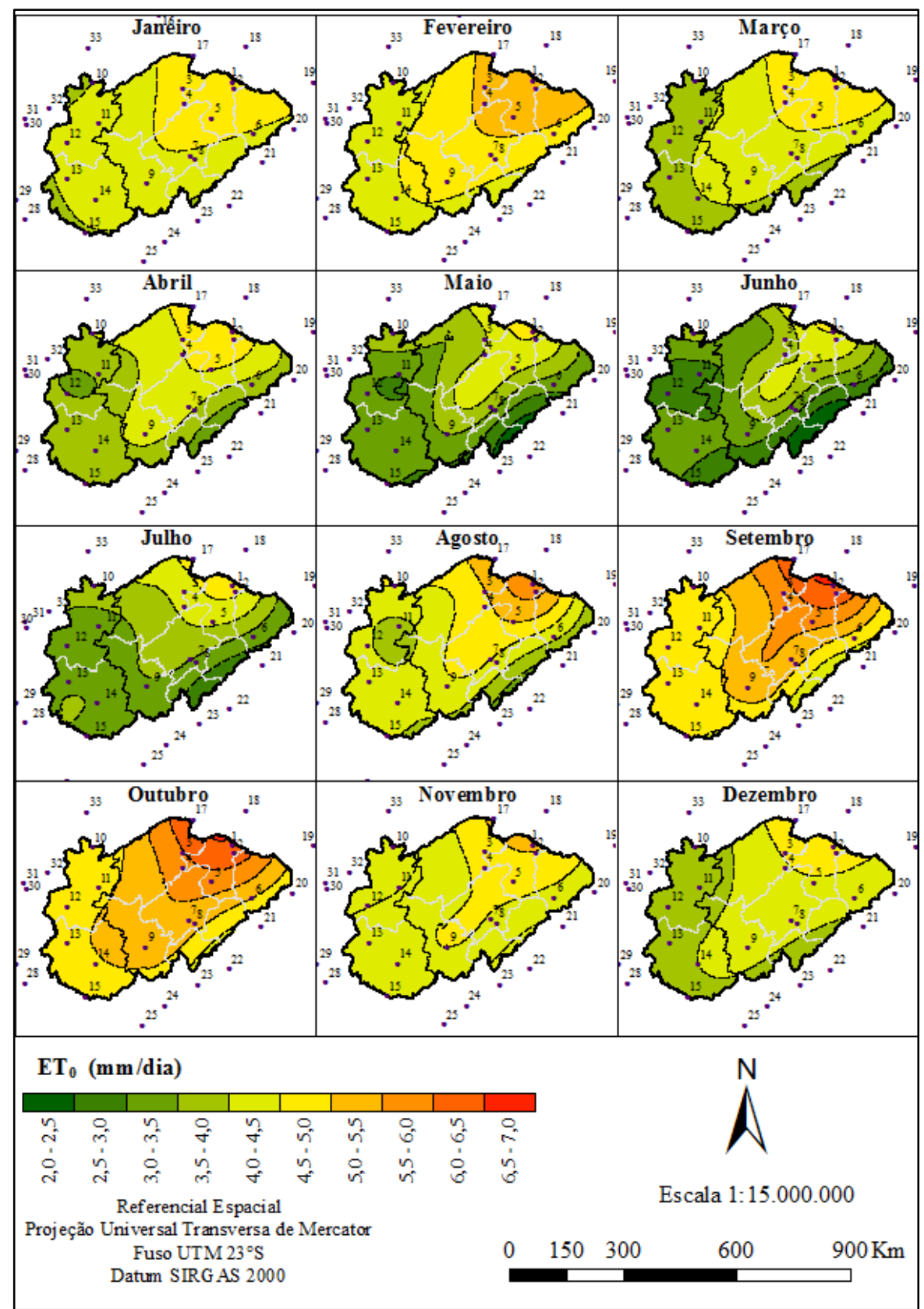

Figura 3. Distribuição espacial de $\mathrm{ET}_{0}$ para a mesorregião do Norte e Noroeste, do estado de Minas Gerais.

\section{Zonas homogêneas}

Os mapas de distribuição espacial de $\mathrm{ET}_{0}$, juntamente com o Balanço Hídrico Climatológico, reforçaram a constatação de que os meses de julho, agosto, setembro e outubro são os mais críticos para o manejo da irrigação na região em estudo, pois a medida que a demanda evapotranspirométricas é crescente, as deficiências hídricas são mais elevadas. Silva Junior et al. (2018) determinaram os meses de agosto, setembro e outubro os meses mais críticos para a agricultura irrigada na região Noroeste de São Paulo.

Os mapas dos meses mais críticos foram sobrepostos para visualização do esboço das zonas homogêneas de evapotranspiração de referência $\left(\mathrm{ET}_{0}\right)$, conforme indicado na Figura 4. 


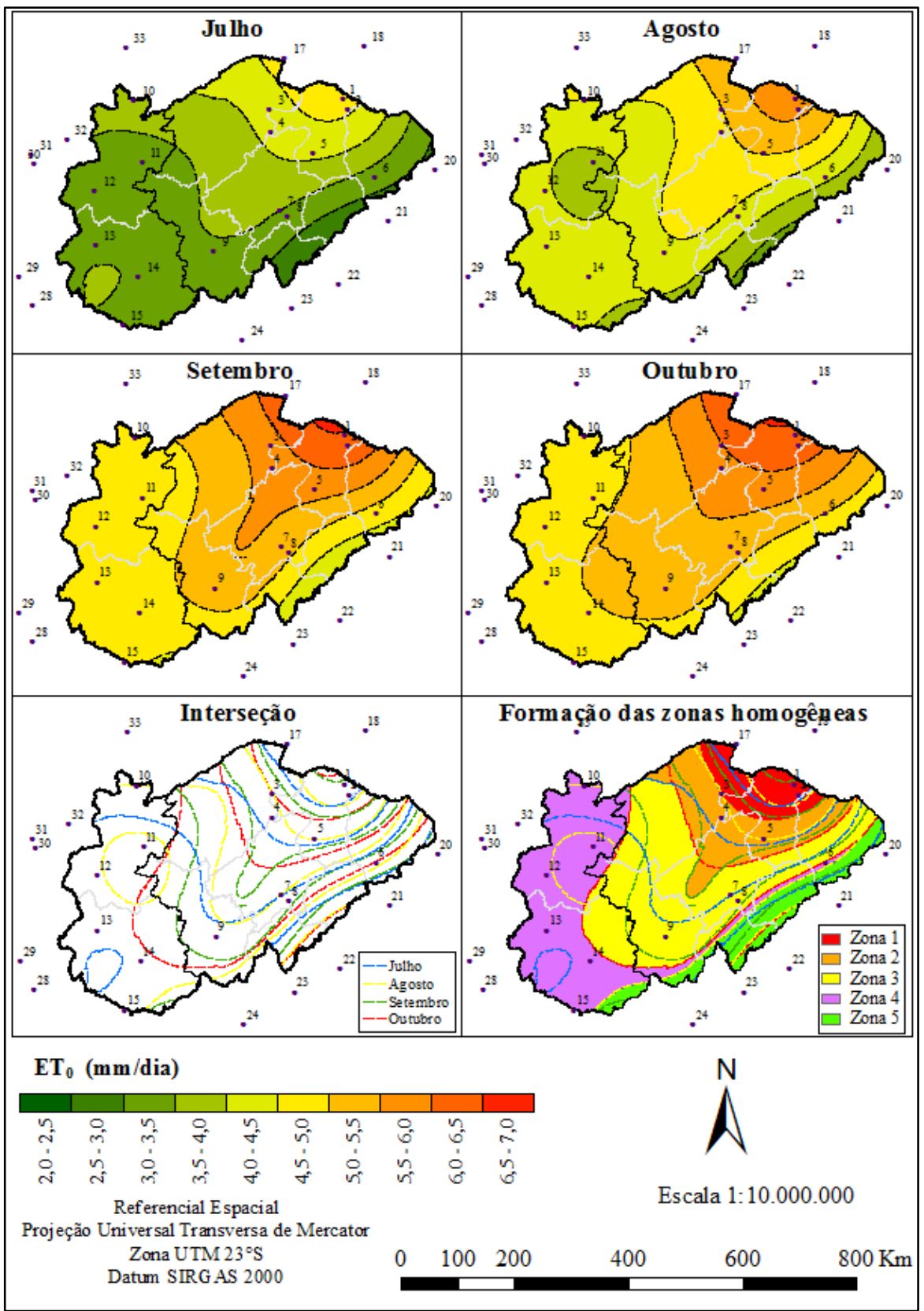

Figura 4. Sobreposição dos mapas para os meses mais críticos para o manejo da irrigação para a mesorregião do Norte e Noroeste, do estado de Minas Gerais.

Foram identificadas cinco zonas homogêneas de $\mathrm{ET}_{0}$ para as mesorregiões Norte e Noroeste do estado de Minas Gerais (Figura 5). A região Noroeste foi abrangida por 3 zonas com as menores taxas evapotranspirométricas, enquanto a região
Norte foi caracterizada por todas as zonas, sendo que mais ao norte registraram-se as zonas de maior demanda evapotranspirométricas e ao sul com a diminuição da demanda. 


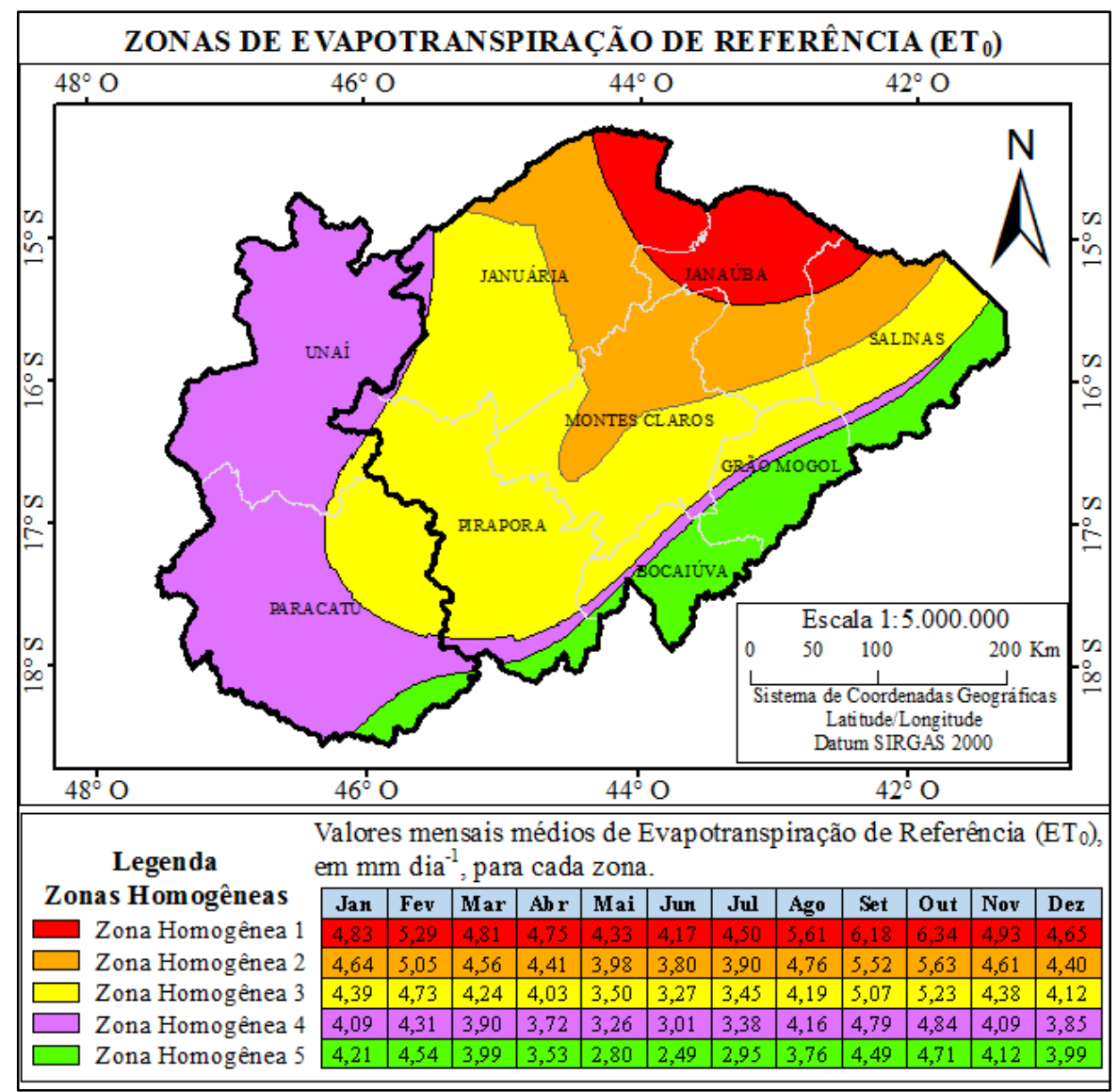

Figura 5. Mapa representativo das Zonas Homogêneas e valores estimados mensais da evapotranspiração de referência $\left(\mathrm{mm} \mathrm{dia}^{-1}\right)$, para cada zona na mesorregião do Norte e Noroeste, do estado de Minas Gerais.

Os valores médios mensais de $\mathrm{ET}_{0}(\mathrm{~mm}$ $\left.\operatorname{dia}^{-1}\right)$, estimados com base na média de cada zona predita pela Krigagem ordinária são apresentados na Tabela 4. Esses valores médios, estimados com base na série histórica de 32 anos, poderão servir como uma ferramenta auxiliar no manejo racional da irrigação.

Tabela 4. Valores mensais médios de evapotranspiração de referência $\left(\mathrm{mm} \mathrm{dia}^{-1}\right)$, para cada zona da mesorregião do Norte e Noroeste, do estado de Minas Gerais.

\begin{tabular}{ccccccccccccc}
\hline Zona & Jan & Fev & Mar & Abr & Mai & Jun & Jul & Ago & Set & Out & Nov & Dez \\
\hline Z1 & 4,83 & 5,29 & 4,81 & 4,75 & 4,33 & 4,17 & 4,50 & 5,61 & 6,18 & 6,34 & 4,93 & 4,65 \\
Z2 & 4,64 & 5,05 & 4,56 & 4,41 & 3,98 & 3,80 & 3,90 & 4,76 & 5,52 & 5,63 & 4,61 & 4,40 \\
Z3 & 4,39 & 4,73 & 4,24 & 4,03 & 3,50 & 3,27 & 3,45 & 4,19 & 5,07 & 5,23 & 4,38 & 4,12 \\
Z4 & 4,09 & 4,31 & 3,90 & 3,72 & 3,26 & 3,01 & 3,38 & 4,16 & 4,79 & 4,84 & 4,09 & 3,85 \\
Z5 & 4,21 & 4,54 & 3,99 & 3,53 & 2,80 & 2,49 & 2,95 & 3,76 & 4,49 & 4,71 & 4,12 & 3,99
\end{tabular}

*Z1 - Zona homogênea 1; Z2 - Zona homogênea 2; Z3 - Zona homogênea 3; Z4 - Zona homogênea 4; Z5 - Zona homogênea 5 .

A zona homogênea 1, em termos de área, foi a menor zona identificada. Localizada mais ao norte das mesorregiões do Norte de Minas, abrangeu partes das microrregiões de Janaúba (54\%), Januária (15\%), Salinas (7\%) e Montes Claros (1\%). O menor valor de $\mathrm{ET}_{0}$ foi observado em junho (média de $4,17 \mathrm{~mm}$ $\operatorname{dia}^{-1}$ ) e o maior valor em outubro (média de $6,34 \mathrm{~mm} \mathrm{dia}^{-1}$ ). Nesta zona homogênea, está inserida a maior parte do projeto público de 
irrigação de Jaíba. Também foram identificados 189 pivôs centrais, irrigando uma área de aproximadamente 12.850 hectares (ANA; EMBRAPA, 2016).

A zona homogênea 2 apresentou a terceira maior área das zonas identificadas. Localizada ao sul da zona homogênea 1, na mesorregião do Norte de Minas, abrangeu partes das microrregiões de Montes Claros (41\%), Januária (33\%), Janaúba (38\%) e Salinas (27\%). Assim como na zona homogênea 1 , o mês de junho apresentou o menor valor $\mathrm{ET}_{0}$ (média de $3,80 \mathrm{~mm} \mathrm{dia}^{-1}$ ) e o mês de outubro, o maior (média de $5,63 \mathrm{~mm}$ $\left.\operatorname{dia}^{-1}\right)$. Nesta zona homogênea está inserido o projeto público de irrigação de Gorutuba, Lagoa Grande e parte do Projeto Jaíba. Nesta zona, foram identificados 206 pivôs centrais, irrigando uma área de aproximadamente 12.709 hectares (ANA; EMBRAPA, 2016).

A zona homogênea 3 apresentou a maior área. Abrangeu partes das microrregiões de Pirapora (83\%), Montes Claros (57\%), Januária (48\%), Salinas (38\%), Grão Mogol (28\%), Bocaiúva (24\%), Paracatu (23\%), Janaúba (8\%) e Unaí (3\%). A maior demanda evapotranspirométricas foi observada em outubro (média de $5,23 \mathrm{~mm} \mathrm{dia}^{-1}$ ) e a menor em junho (média de $3,27 \mathrm{~mm} \mathrm{dia}^{-1}$ ). Estão inseridos nesta zona homogênea, o projeto público de irrigação de Pirapora e extensas áreas irrigadas por pivô central nos municípios de São Romão, Jequitaí, Várzea da Palma e Buritizeiro. Foram identificados 562 pivôs centrais, irrigando uma área de aproximadamente 45.298 hectares (ANA; EMBRAPA, 2016).

A zona homogênea 4 foi a segunda maior zona identificada. Essa se localiza majoritariamente, a oeste da mesorregião
Noroeste. Compreende partes das microrregiões de Unaí (97\%), Paracatu (71\%), Bocaiúva (11\%), Grão Mogol (11\%), Pirapora (11\%), Januária (4\%), Salinas (4\%) e Montes Claros (1\%). Também nesta zona homogênea, a maior demanda evapotranspirométricas foi observada em outubro (média de 4,84 $\mathrm{mm}$ dia ${ }^{1}$ ) e menor em junho (média de $3,01 \mathrm{~mm} \mathrm{dia}^{-1}$ ). Nesta zona homogênea estão inseridas as maiores áreas irrigadas por pivô central, situadas nos municípios de Unaí, Paracatu, Buritis, Formoso, Guarda-Mor, Presidente Olegário, Vazante, Lagamar, Lagoa Grande e João Pinheiro. Foram identificados 2.341 pivôs centrais, irrigando uma área de aproximadamente 170.747 hectares (ANA; EMBRAPA, 2016).

A zona homogênea 5, a segunda menor zona identificada, está localizada ao sul das mesorregiões Norte e Noroeste de Minas, e abrangeu partes das microrregiões de Bocaiúva (65\%), Grão Mogol (61\%), Salinas (24\%), Paracatu (6\%), Pirapora (7\%). O menor valor de $\mathrm{ET}_{0}$ foi observado em junho (média de 2,49 $\mathrm{mm} \mathrm{dia}^{-1}$ ) e o maior em outubro (média de 4,71 mm $\mathrm{dia}^{-1}$ ). Na zona homogênea 5 estão inseridas áreas irrigadas por pivô central situadas nos municípios Bocaiúva e Águas Vermelhas, principalmente. Foram identificados 36 pivôs centrais, irrigando uma área de aproximadamente 2.229 hectares (ANA; EMBRAPA, 2016).

Para a validação das zonas homogêneas, os valores preditos pela Krigagem de $\mathrm{ET}_{0}$ foram correlacionados com os valores mensais de $\mathrm{ET}_{0}\left(\mathrm{~mm} \mathrm{dia}{ }^{-1}\right)$ observados pelas 14 estações meteorológicas convencionais. Os índices analisados são apresentados na Tabela 5. 
Tabela 5. Indicadores utilizados para a validação das zonas homogêneas de $\mathrm{ET}_{0}$ a partir dos dados observados e preditos pela Krigagem ordinária, ajustado para o modelo Gaussiano do semivariograma.

\begin{tabular}{cccccccccc}
\hline Meses & REQM & $\mathrm{d}$ & $\mathrm{r}$ & $\mathrm{c}$ & Desempenho & $\mathrm{R}^{2}$ & EMA & EMP & NSE \\
\hline Janeiro & 0,205 & 0,8808 & 0,7990 & 0,70 & Bom & 0,6384 & 0,154 & 3,508 & 0,459 \\
Fevereiro & 0,273 & 0,8858 & 0,8149 & 0,72 & Bom & 0,6641 & 0,211 & 4,437 & 0,458 \\
Março & 0,208 & 0,9206 & 0,8740 & 0,80 & Muito bom & 0,7639 & 0,177 & 4,195 & 0,487 \\
Abril & 0,181 & 0,9491 & 0,9196 & 0,87 & Ótimo & 0,8457 & 0,158 & 3,963 & 0,589 \\
Maio & 0,221 & 0,9362 & 0,9002 & 0,84 & Muito bom & 0,8104 & 0,173 & 4,781 & 0,594 \\
Junho & 0,240 & 0,9373 & 0,9046 & 0,85 & Muito bom & 0,8183 & 0,191 & 5,552 & 0,587 \\
Julho & 0,275 & 0,9078 & 0,8745 & 0,79 & Muito bom & 0,7647 & 0,218 & 5,773 & 0,508 \\
Agosto & 0,366 & 0,8951 & 0,8408 & 0,75 & Muito bom & 0,7070 & 0,301 & 6,511 & 0,404 \\
Setembro & 0,416 & 0,8693 & 0,8297 & 0,72 & Bom & 0,6885 & 0,371 & 6,879 & 0,308 \\
Outubro & 0,385 & 0,8840 & 0,8174 & 0,72 & Bom & 0,6682 & 0,343 & 6,385 & 0,315 \\
Novembro & 0,201 & 0,9092 & 0,8663 & 0,79 & Muito bom & 0,7505 & 0,171 & 3,841 & 0,460 \\
Dezembro & 0,237 & 0,8665 & 0,7800 & 0,68 & Bom & 0,6085 & 0,190 & 4,579 & 0,383 \\
\hline
\end{tabular}

* $\mathrm{R}^{2}$ - Coeficiente de determinação da validação cruzada; REMQ - Raiz do Erro Médio Quadrático; d - Índice de Concordância; r - Coeficiente de Correlação de Pearson; c - Índice de Confiança; EMA - Erro Médio Absoluto (mm dia ${ }^{1}$ ); EMP - Erro Médio Percentual; e NSE - Eficiência Nash-Sutcliffe.

Segundo Souza et al. (2011), o emprego desse conjunto de indicadores estatísticos pode ser considerado uma boa opção para validação de modelos estatísticos. Os indicadores permitem a realização de análises simultâneas do desvio da média e da identificação da ocorrência de sub ou superestimativa, espalhamento e ajustamento dos modelos, quando comparados com valores estimados e medidos (SILVA JUNIOR, 2017).

$\mathrm{O}$ índice de concordância (d) refletiu a precisão dos valores de $\mathrm{ET}_{0}$ preditos pela Krigagem ordinária em relação aos valores observados pelas estações, o valor médio foi de 0,9030 (variando de 0,8665 à 0,9491), indicando a elevada concordância entre os valores estimados e interpolados. Os valores obtidos de "r" indicam que a krigagem interpolou a $\mathrm{ET}_{0}$ com elevada correlação (média de 0,852). Já os valores de " $R$ ", encontrados (média de 0,727 ) indicam que os valores estimados e interpolados variaram de forma proporcional, que demonstra de forma satisfatória a variabilidade sazonal da $\mathrm{ET}_{0}$ (SILVA JUNIOR, 2017). Os valores de REMQ variaram de 0,181 à 0,416 , com média de 0,267. Os resultados encontrados estão similares aos encontrados por Vila Nova, et. al. (2012) e por Silva Junior (2017).
Analisando conjuntamente a precisão e a exatidão dos resultados obtidos, através do índice de confiança (c), o desempenho foi considerado bom para 5 meses, muito bom para 6 meses e ótimo para um mês, segundo critério de Camargo e Sentelhas (1997). Ressalta-se que nos entre os meses março a agosto, foi o período onde observou os melhores índices.

O erro médio absoluto (EMA) variou de $0,154 \mathrm{~mm} \mathrm{dia}^{-1}$, em janeiro até $0,371 \mathrm{~mm} \mathrm{dia}^{-1}$ em setembro. Já o erro médio percentual (EMP) variou de 3,5\% em janeiro até 6,9\% em outubro.

O coeficiente de eficiência de NashSutcliffe (NSE) varia entre $-\infty$ e 1 , sendo que valores inferiores a zero acusam uma ineficiência do método de interpolação. Quanto maior o valor, melhor a eficiência do método. O índice NSE variou de 0,308 a 0,594, com valor médio de 0,463, demonstrando a eficiência no método de interpolação (krigagem) utilizado no trabalho.

Os resultados das estimativas de $\mathrm{ET}_{0}$ obtidos via krigagem, baseando-se nos indicadores expostos na Tabela 5, foram considerados satisfatórios. Resultados semelhantes também foram encontrados por Hodam et al. (2017). 


\section{CONCLUSÕES}

Foram identificadas 5 zonas homogêneas de $\mathrm{ET}_{0}$ para as mesorregiões Norte e Noroeste do estado de Minas Gerais. As médias de evapotranspiração preditas pela Krigagem ordinária foram similares às observadas (medidas) pelas estações meteorológicas convencionais. Os indicadores estatísticos mostraram-se satisfatórios. Esses valores estimados de $\mathrm{ET}_{0}$ podem ser utilizados como informação auxiliar na elaboração de projetos e no manejo da irrigação.

\section{REFERÊNCIAS BIBLIOGRÁFICAS}

ANA - Agência Nacional de Águas; EMBRAPA Milho e Sorgo. Levantamento da agricultura irrigada por pivôs centrais no Brasil: ano 2014. Brasília, 2016. 1 mapa. Escala 1:50.000 Disponível em: <http://www.ana.gov.br/metadados>. Acesso em: 15 jun. 2017.

ALLEN, R. G.; PEREIRA, L. S.; RAES, D.; SMITH, M. Crop evapotranspiration: Guidelines for computing crop water requirements. Roma: FAO, 1998. (FAO Irrigation and Drainage Paper n. 56.)

BEZERRA, B. G.; SILVA, B. B.; FERREIRA, N. J. Estimativa da evapotranspiração real diária utilizando-se imagens digitais TM - Landsat 5. Revista Brasileira de Meteorologia, v. 23, n. 3, p. 305-317, 2008.

CAMARGO, A. P.; SENTELHAS, P. C. Avaliação do desempenho de diferentes métodos de estimativa da evapotranspiração potencial no estado de São Paulo, Brasil. Revista Brasileira de Agrometeorologia, v. 5, n. 1, p. 89-97, 1997.

CASTRO, F. da S.; PEZZOPANE, J. E. M.; CECÍlIO, R. A.; PEZZOPANE, J. R. M.; XAVIER, A. C. Avaliação do desempenho dos diferentes métodos de interpoladores para parâmetros do balanço hídrico climatológico. Revista Brasileira de Engenharia Agrícola e
Ambiental, v. 14, n. 8, p.871-880, 2010.

GAMMA DESIGN SOFTWARE. GS+TMGeoStatics for the Environmental Sciences. Version 10 Demo. Plainwell, Michigan: Gamma Design Software, 2002.

HODAM, S.; SARKAR, S.; MARAK, A. G. R.; BANDYOPADHYAY, A.; BHADRA, A. Spatial Interpolation of Reference Evapotranspiration in India: Comparison of IDW and Kriging Methods. Journal of The Institution of Engineers, v. 98 , n. 4, p. 511524, 2017.

IBGE - Instituto Brasileiro de Geografia e Estatística. Bases e referenciais. Rio de Janeiro: IBGE, 2016. Vários mapas. Escalas variam. Disponível em: <https://mapas.ibge.gov.br>. Acesso em: 01 jun. 2017.

INMET - Instituto Nacional de Meteorologia. Banco de Dados Meteorológicos para Ensino e Pesquisa (BDMEP). Brasília, DF: INMET, 2017. Disponível em: <http://www.inmet.gov.br>. Acesso em: 15 set. 2017.

INMET - Instituto Nacional de Meteorologia. Normais Climatológicas do Brasil, 19812010. Brasília: INMET, 2018. Disponível em: $<$ http://www.inmet.gov.br/portal/index.php?r= clima/normaisClimatologicas $>$. Acesso em: 12 fev. 2019.

LIANG, L.; LI, L.; LIU, Q. Temporal variation of reference evapotranspiration during 1961-2005 in the Taoer River basin of Northeast China. Agricultural and Forest Meteorology, v. 150, n. 2, p. 298-306, 2009.

MANCOSU, N.; SNYDER, R. L.; SPANO, D. Procedures to Develop a Standardized Reference Evapotranspiration Zone Map. Journal of Irrigation and Drainage Engineering, v. 140, n. 9, 2014. https://doi.org/10.1061/(ASCE)IR.19434774.0 000697 
MOTOMIYA, A. V. A.; CORÁ, J. E.; PEREIRA, G. T. Uso da krigagem indicatriz na avaliação de indicadores de fertilidade do solo. Revista Brasileira de Ciência de Solo, v. 30, p. 485-496, 2006.

NASH, J. E.; SUTCLIFFE, J. V. River flow forecasting through conceptual models, Part I A discussion of principles, Journal of Hydrology, v. 10, n. 3, p - 282-290, 1970.

PEREIRA, L. S., ALLEN, R. G., SMITH, M., RAES, D. Crop evapotranspiration estimation with FAO56: Past and future. Agricultural Water Management, v. 147, p. 4-20, 2015.

PIRES, C. A. F.; REIS, J. T.; DULLIUS, Â. I; S.; PRIESNIT, M. C.; PEREIRA, M. J. Krigagem ordinária aplicada à precipitação pluviométrica nos estados do Rio Grande do Sul e Santa Catarina. Ciência e Natura, v. 38, n. 3, p. $1303-1311,2016$.

ROLIM, G. S.; SENTELHAS, P. C; BARBIERI, V. Planilhas no ambiente EXCEL TM para os cálculos de balanços hídricos: normal, sequencial, de cultura e de produtividade real e potencial. Revista Brasileira de Agrometeorologia, v. 6, n. 1, p. 133-137, 1998.

SANCHES, F. M.; XIMENES, A. R.; CORADI, P. C.; ROQUE, C. G.; CUNHA, F. F.. Estimativa da evapotranspiração de referência na região Norte do Brasil. Revista de Ciências Agroambientais, v. 13, n. 2, p. 19-31, 2015.

SILVA JUNIOR, J. F. Evapotranspiração de referência como base para o manejo sustentável da irrigação no Noroeste Paulista. 2017. 82 f. Tese (Doutorado em Agronomia) - Faculdade de Ciências Agronômicas da UNESP, Botucatu.

SILVA JUNIOR, J. F.; HERNANDEZ, F. B. T.; SILVA, I. P. F.; REIS, L. S.; TEIXEIRA, A. H. de C. Estabelecimento dos meses mais críticos para a agricultura irrigada a partir do estudo do balanço hídrico. Brazilian Journal of Biosystems Engineering, v. 12, n. 2, p. 122-131, 2018.

SOUZA, A. P. de; CARVALHO, D. F. de; SILVA, L. B. D. da; ALMEIDA, F. T. de; ROCHA, H. S. Estimativas da evapotranspiração de referência em diferentes condições de nebulosidade. Pesquisa Agropecuária Brasileira, v. 46, p. 219-228, 2011.

THORNTHWAITE, C. W.; MATHER, J. R. The water balance. New Jersey: Drexel Institute of Technology, 1955. $104 \mathrm{p}$.

VIANELLO, R. L.; ALVES, A. R. Meteorologia básica e aplicações. 2. ed. Viçosa: Editora UFV, 2012. 460 p.

VICENTE, M. R.; MANTOVANI, E. C.; FERNANDES, A. L. T.; DELAZARI, F. T.; FIGUEREDO, E. M. Efeito de diferentes lâminas de irrigação nas variáveis de desenvolvimento e produção do cafeeiro irrigado por pivô central. Irriga, v. 20, n. 3, p. 528-543, 2015.

VICENTE, M. R.; SANTOS, J. A.; SANTOS, R. M.; LEITE, C. V. Comparação entre métodos de estimativa da evapotranspiração de referência para o Vale do Jequitinhonha. Global Science and Technology, v. 07, n. 02, 2014. p.106 - 118 .

VILA NOVA, M. R. N.; SIMÕES, S. J. C.; TRANNIN, I. C, B. Interpolação geoespacial da evapotranspiração de referência (ETo) em regiões com escassez de dados: estudo de caso no Sul de Minas Gerais. Revista Ambiente \& Água, v. 7, n. 2, p. 179-194, 2012. http://dx.doi.org/10.4136/ambi-agua.763.

WILLMOTT, C. J. On the validation of models. Physical Geography, v. 2, p. 184 194, 1981. 